\title{
DIAGNÓSTICO DE MALFORMAC̣ÃO ARTERIOVENOSA UTERINA POR MEIO DA ULTRA-SONOGRAFIA COM DOPPLER COLORIDO E ACHADOS À ANGIORRESSONÂNCIA MAGNÉTICA: RELATO DE CASO*
}

Ayrton Roberto Pastore ${ }^{1}$, Daniel Pastore ${ }^{2}$, Francisco César Carnevale ${ }^{3}$, Airton Mota Moreira ${ }^{4}$, Alice Kano ${ }^{5}$, Giovanni Guido Cerri ${ }^{6}$

Resumo As malformações arteriovenosas do útero são entidades raras. Sua forma de apresentação clínica é muito diversa, devendo o ginecologista e o imaginologista estar atentos para esta possibilidade diagnóstica, para estabelecer o tratamento de forma precisa e rápida. 0 presente artigo visa mostrar um caso de malformação arteriovenosa uterina adquirida após doença trofoblástica gestacional, cujo diagnóstico foi bem estabelecido por meio da ultra-sonografia com Doppler colorido e correlação com angiorressonância magnética. Unitermos: Angiografia; Doppler; Embolização; Ultra-sonografia; Útero; Malformação vascular.

Abstract Diagnosis of uterine arteriovenous malformation by Doppler ultrasonography and magnetic angioresonance findings: a case report.

Uterine arteriovenous malformations are rare and the clinical features quite variable. Gynecologists and radiologists must be aware of this diagnostic possibility in order to indicate a fast and accurate treatment. The aim of the present paper is to present a case of uterine arteriovenous malformation acquired after trophoblastic gestational disease for which diagnosis was well established by means of color Doppler ultrasonography and correlation to magnetic angioresonance.

Key words: Angiography; Doppler; Embolization; Ultrasonography; Uterus; Vascular malformation.

\section{INTRODUÇÃO}

A malformação arteriovenosa (MAV) uterina é uma condição rara, cuja incidência verdadeira ainda não é bem conhecida. $\mathrm{Na}$ atualidade, apenas 26 casos foram relatados na literatura ${ }^{(\mathbf{1})}$. A doença recebe, também, outras denominações: fístula arteriovenosa, angioma cavernoso, angioma pulsátil, arteriectasia e aneurisma cirsóide. Esta pode ser adquirida ou congênita ${ }^{(2,3)}$.

Relatamos um caso de MAV uterina diagnosticada com sucesso por meio da ul-

* Trabalho realizado no Instituto de Radiologia (InRad) do Hospital das Clínicas da Faculdade de Medicina da Universidade de São Paulo (HC-FMUSP), São Paulo, SP.

1. Assistente Doutor do InRad/HC-FMUSP.

2. Médico Residente do Departamento de Radiologia do Hospital de Base da Faculdade de Medicina de São José do Rio Preto.

3. Médico Chefe do Serviço de Radiologia Intervencionista do HC-FMUSP.

4. Médico Assistente do Serviço de Radiologia Intervencionista do HC-FMUSP.

5. Médica Assistente da Clínica Obstétrica do Hospital do Servidor Municipal de São Paulo.

6. Professor Titular do Departamento de Radiologia da FMUSP.

Endereço para correspondência: Dr. Ayrton Roberto Pastore. Rua Capital Federal, 827, Sumaré. São Paulo, SP, 01259-010. E-mail: arpastore@ajato.com.br

Recebido para publicação em 22/9/2003. Aceito, após revisão, em 22/10/2003. tra-sonografia transvaginal com Doppler colorido e correlação com angiorressonância magnética.

\section{RELATO DO CASO}

Paciente com 36 anos de idade, branca, nulípara, natural de Andradina, SP. Encaminhada para consulta clínica de rotina, relatando história de sangramento genital intermitente e profuso há cerca de sete anos. Há oito anos, realizou duas curetagens uterinas por hipótese de abortamento incompleto (no intervalo de um mês), com anatomopatológico de restos ovulares (sic), apresentando persistência do sangramento e níveis sanguíneos elevados da fração-beta da gonadotrofina coriônica humana ( $\beta$-hCG), de $105.000 \mathrm{UI} / \mathrm{L}$, sendo levantada a hipótese diagnóstica de doença trofoblástica gestacional (mola invasora ou coriocarcinoma).

Foi submetida a tratamento quimioterápico, com negativação dos títulos de $\beta$ hCG após seis meses. Dentro de um ano, a paciente iniciou quadro de metrorragias (sangramentos profusos e intermitentes) e títulos de $\beta$-hCG para pesquisa de doença molar indetectáveis, sendo submetida a vídeo-histeroscopia associada a laparoscopia, com biópsia de endométrio (endométrio em fase proliferativa intermediária) e biópsia de miométrio (miométrio normal, sem vestígios de doença molar ou endometriose). Há cerca de três anos vem sendo tratada com interferon (sorologia positiva para hepatite $\mathrm{C}$ ), com controle estável e negativação do vírus (função hepática normal). Até o momento, vinha se mantendo em amenorréia por uso de contraceptivos orais combinados de uso contínuo de alta dosagem (etinil estradiol $50 \mu \mathrm{g}$ e Levonorgestrel $\left.^{\circledR} 250 \mu \mathrm{g}\right)$, porém, em virtude do passado de hepatite e da necessidade de troca de medicamento que não interferisse na passagem hepática, foi sugerida a suspensão dos contraceptivos orais, e devido à não adaptação aos contraceptivos de uso percutâneo ou endoceptivo, a paciente teve novos quadros de metrorragias.

Foi realizado exame de ultra-sonografia transvaginal, que mostrou útero aumentado de volume, com alterações texturais difusas heterogênea associadas a múltiplas 
imagens císticas de diferentes tamanhos no interior do miométrio (Figuras 1 e 2). O estudo pelo Doppler colorido convencional (Figura 3) e de amplitude (Figura 4) evidenciou padrão de acentuada vascularização uterina, principalmente em parede posterior, acometendo desde a região cervical até o fundo uterino, com vasos dilatados medindo cerca de $2,0 \mathrm{~cm}$, indicando "shunt" arteriovenoso (fluxo arterial e venoso). O Doppler pulsado mostrou padrão de baixo índice de resistência nos vasos da amostra (Figura 5). O exame de angiorressonância magnética demonstrou a MAV uterina, caracterizada em imagens "spinecho" convencionais nas sequiências ponderadas em T1 e T2 por múltiplos "flowvoids" em topografia miometrial (Figuras

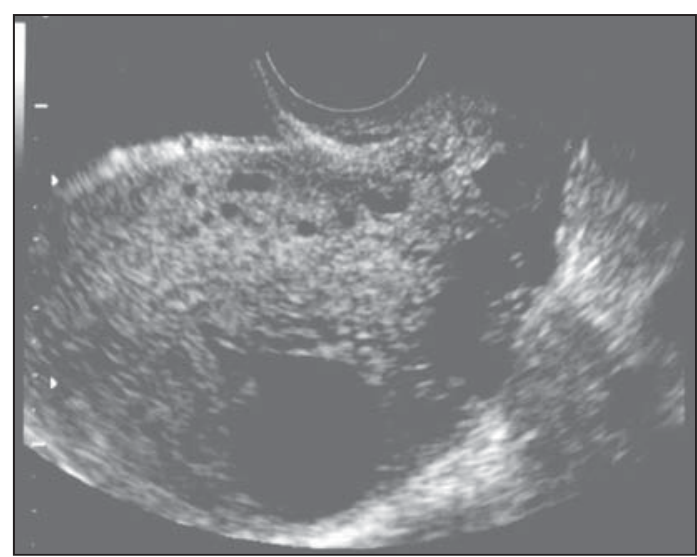

Figura 1. Ultra-sonografia transvaginal, corte sagital. As formações vasculares são identificadas desde a região cenvical até a região corporal posterior, como formações anecogênicas circulares e tubulares, medindo a maior $2,0 \mathrm{~cm}$ no maior diâmetro. 0 miométrio apresenta alteração textural difusa.

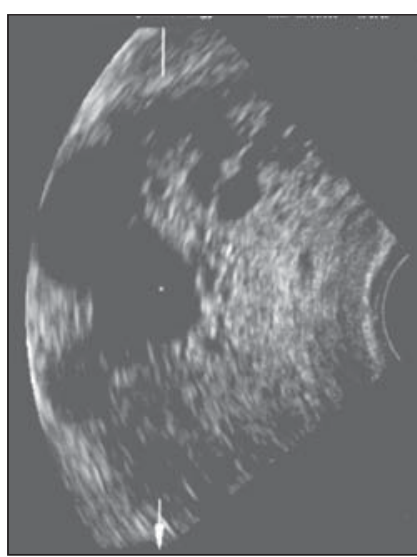

Figura 2. Ultra-sonografia tridimensional, corte coronal (frontal). Neste corte é possível identificar a disposição dos vasos miometriais e o aspecto serpiginoso e dilatado (aneurismático).

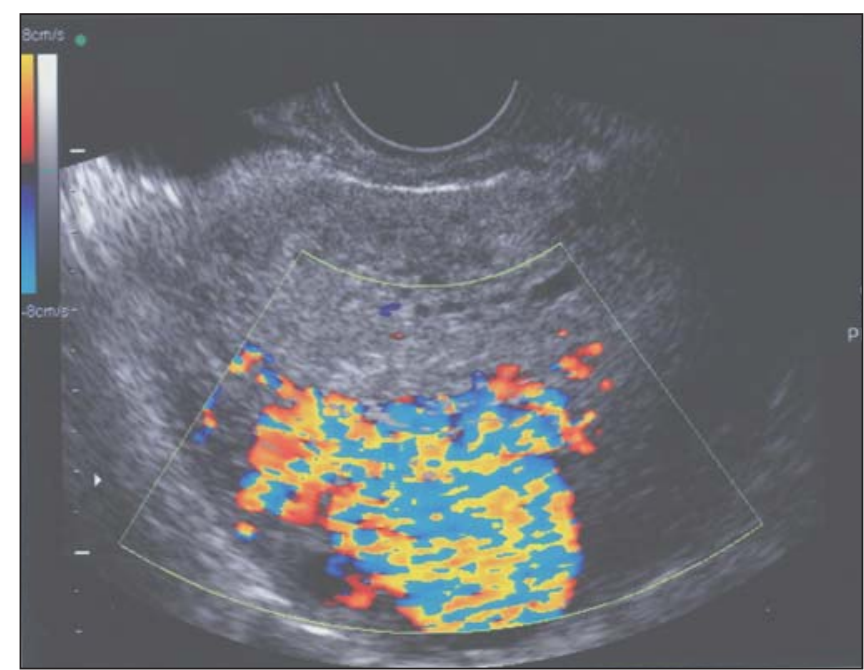

Figura 3. A ultra-sonografia com Doppler colorido convencional identifica o fluxo sanguíneo com "aliasing" no miométrio.

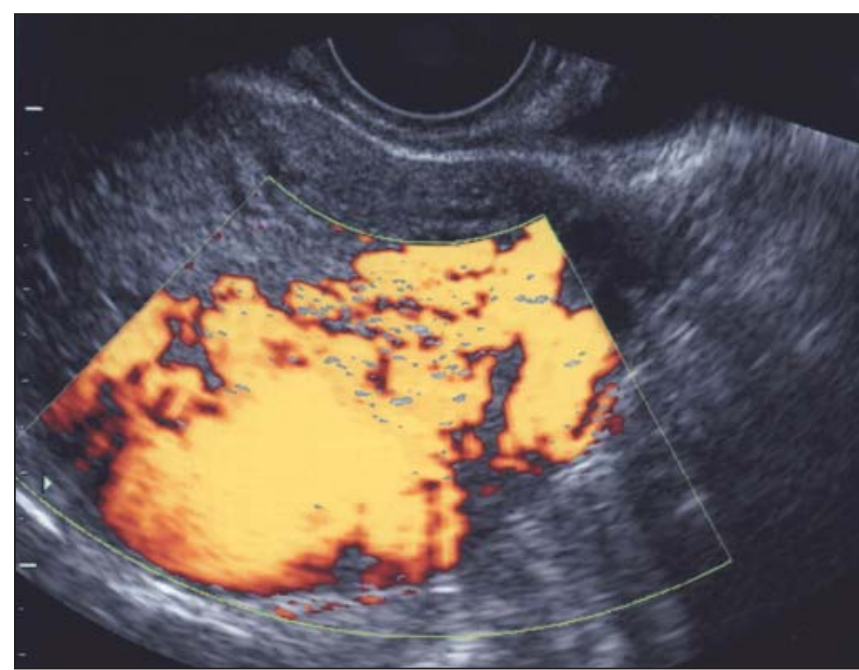

Figura 4. Aspecto da malformação arteriovenosa à ultra-sonografia com Doppler colorido de amplitude.
6 e 7). Neste caso visualizava-se volumosa massa na escavação pélvica apresentando sinal intenso, aparentemente nutrida pelos ramos da artéria ilíaca interna direita, com drenagem venosa anômala rápida, através de veia calibrosa que se estende cranialmente, anterior à lesão, caracterizando a MAV (Figuras 8 e 9).

No momento, a paciente encontra-se em acompanhamento para estudo angiográfico e possível embolização.

\section{DISCUSSÃO}

A MAV uterina foi primeiramente descrita em 1926, por Dubreil e Loubat ${ }^{(2)}$,

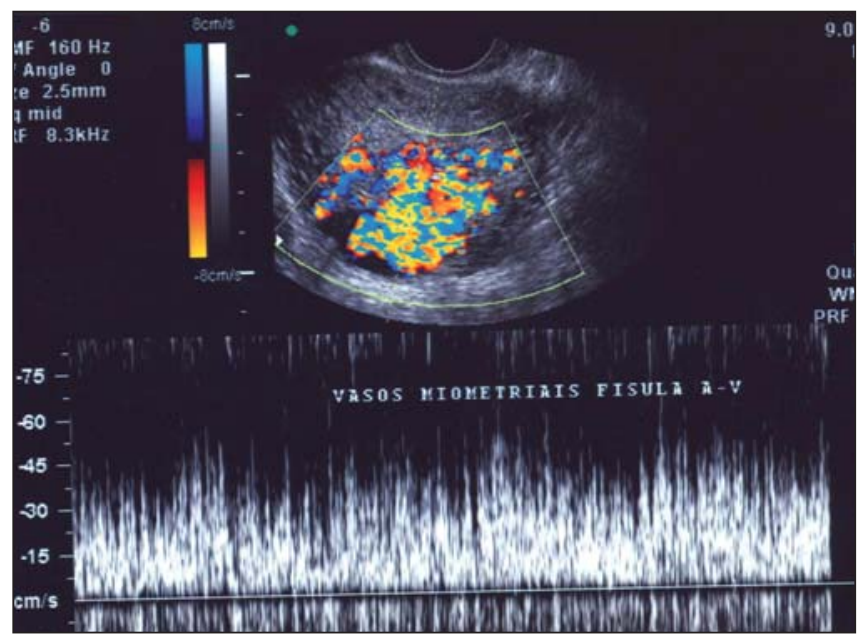

Figura 5. O Doppler pulsado dos vasos miometriais mostra o padrão espectral de fluxo de baixa impedância com ruído, decorrente da presença de fístulas arteriovenosas. 


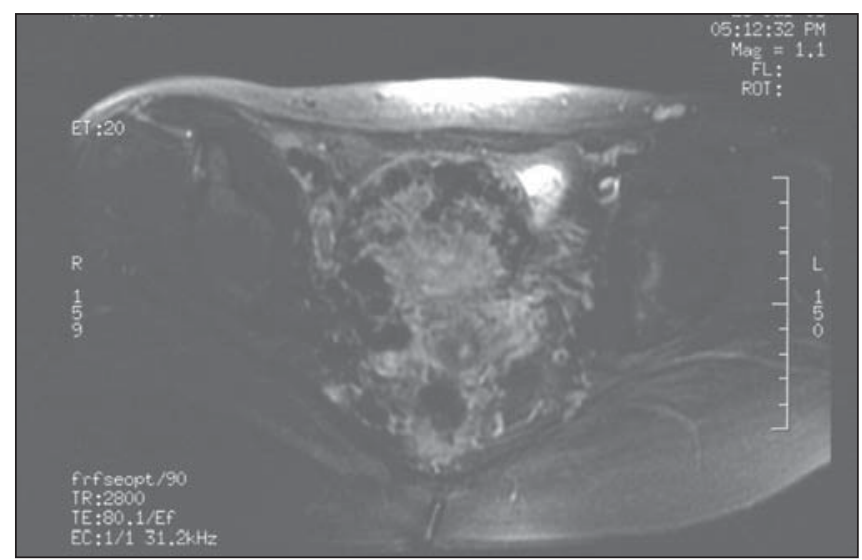

Figura 6. Imagem em T2 com saturação de gordura. Observa-se, no plano da escavação pélvica, grande massa mal delimitada, de contornos bocelados, apresentando múltiplas imagens de abolição do sinal ("flow void") associadas a vasos com velocidade de fluxo aumentada. Notar também pequeno foco de hipersinal correspondendo ao endométrio, canal cervical e vasos calibrosos na espessura do miométrio.

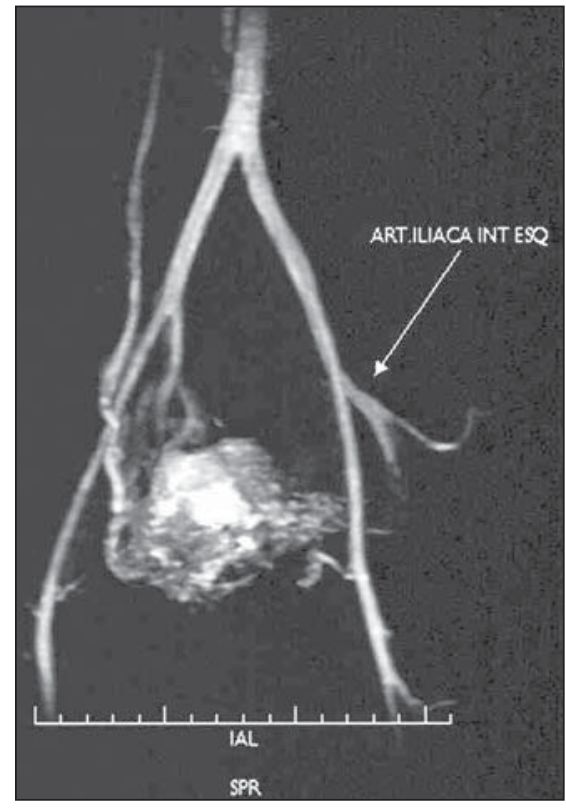

Figura 8. Imagem da aorta e vasos ilíacos, em projeção de intensidade máxima. Observa-se contrastação adequada da aorta e artérias ilíacas comuns, externas e internas, com volumosa massa apresentando sinal intenso, aparentemente nutrida pelos ramos da artéria ilíaca interna direita, com drenagem venosa anômala rápida pela veia calibrosa, que se estende cranialmente, anterior à lesão.

como aneurisma cirsóide. Embora as malformações possam ser congênitas ou adquiridas, a grande maioria delas é adquirida. A forma congênita é extremamente rara e resulta de um defeito na diferenciação vascular embrionária ou do desenvolvimento prematuro de um plexo capilar, levando a múltiplas conexões anormais entre artérias

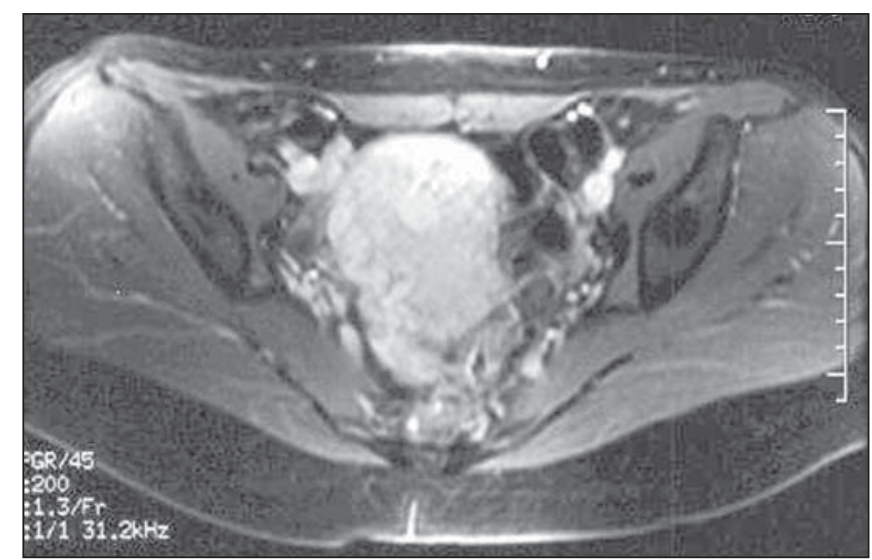

Figura 7. Imagem axial em T1 pós-injeção de contraste paramagnético (gadolínio). Observa-se imagem uterina de contornos bocelados apresentando vaso calibroso na parede póstero-lateral direita, tortuoso, associado a vaso de aspecto venoso junto à região cornual direita, medial aos vasos ilíacos. (Comparar com a ultra-sonografia tridimensional - Figura 2).

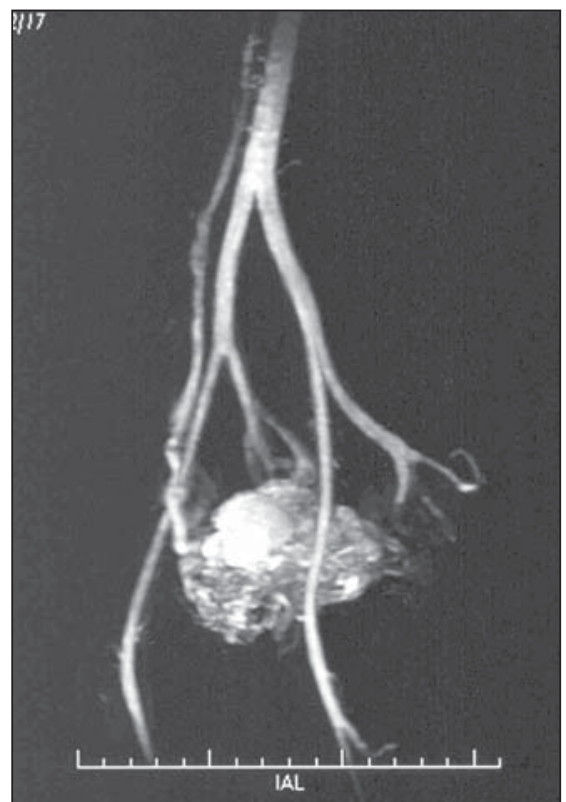

Figura 9. Seqüência de angiorressonância com projeção de intensidade máxima, em rotação. Observam-se, nesta projeção, os mesmos achados do exame anterior, com enchimento precoce da lesão ricamente vascularizada, por ramos da artéria ilíaca interna direita e drenagem anômala venosa ascendente por veia calibrosa ântero-lateral direita (veia gonadal direita).

e veias ${ }^{(3,4)}$. Entretanto, as MAV são adquiridas após lesão do tecido uterino e resultam de doença trofoblástica, trauma pélvico, abortamento, dilatação e curetagem, cesárea, endometriose, infecção uterina, neoplasia cervical ou endometrial e exposição ao dietiletilbestrol ${ }^{(1,5-11)}$. A grande incidência de fístula arteriovenosa uterina tem sido relatada após surgimento quimioterápico de doença trofoblástica ${ }^{(3,10,12-15)}$.

Apesar de as MAV uterinas adquiridas acometerem pacientes jovens, elas mais comumente atingem mulheres acima dos 30 anos de idade. Os principais sinais e sintomas clínicos são hipermenorréia, anemia e sangramento uterino importante. Dor em baixo ventre é rara. A curetagem pode piorar o sangramento, podendo causar choque e até mesmo a morte, quando o diagnóstico de MAV não é feito antes da intervenção, sendo, portanto, contra-indicada nestes $\operatorname{casos}^{(16)}$.

Vários métodos podem ser utilizados para diagnosticar a MAV uterina, incluindo a ultra-sonografia com Doppler colorido, a tomografia computadorizada com contraste, a ressonância magnética e a angiografia $^{(1,11,15,17-20)}$. A angiografia, atualmente, é reservada para os casos de intervenção cirúrgica ou embolização terapêutica ${ }^{(15,17)}$. As características ultra-sonográficas são inespecíficas e incluem presença de estruturas tubulares, serpiginosas ou císticas, hipoecogênicas no miométrio ${ }^{(21)}$. O exame ultra-sonográfico com o Doppler colorido é mais específico no diagnóstico da MAV, notando-se vasos calibrosos com fluxo reverso. A análise espectral destas lesões mostra fluxo de baixa resistência e alta velocidade ${ }^{(1,17)}$.

A ultra-sonografia com Doppler colorido tem sido útil na diferenciação da hidrossalpinge e outras entidades, em relação às varizes pélvicas. Em nosso caso, a 
utilização do Doppler colorido mostrou ser um método valioso para o diagnóstico da MAV uterina, devido aos achados de hipervascularização miometrial e fluxo reverso e de baixa impedância. Do mesmo modo, a angiorressonância magnética permite fácil diferenciação das lesões vasculares daquelas de origem tumoral ou inflamatória, reafirmando os achados da ultra-sonografia com Doppler colorido.

A embolização é indicada como método terapêutico primário, sendo a angiografia pélvica essencial para o planejamento cirúrgico $^{(10,12,13,15,16)}$. Dependendo dos sintomas, histerectomia ou ligação das artérias uterinas são algumas vezes realizadas, quando a paciente não pretende mais engravidar.

\section{CONSIDERAÇÕES FINAIS}

A utilização da ultra-sonografia transvaginal com Doppler colorido vem mostrando, atualmente, ser o método preferencial no diagnóstico de MAV, e o exame de angiorressonância magnética tem obtido imagens idênticas aos da angiografia, sendo considerado um excelente método não invasivo para o diagnóstico desta doença. Este caso visa estudar as MAV uterinas e pélvicas, e alertar os clínicos e imaginologistas desta possibilidade diagnóstica nos casos de pacientes com história de tratamento prévio de doença trofoblástica gestacional, a fim de se estabelecer tratamento e planejamento terapêutico adequados.

\section{REFERÊNCIAS}

1. Sugiyama T, Honda S, Kataoka A, Komai K, Izumi S, Yakushiji M. Diagnosis of uterine arteriovenous malformation by color and pulsed Doppler ultrasonography. Ultrasound Obstet Gynecol 1996;8: 359-60.

2. Dubreil G, Loubat E. Aneurysme cirsoide de l'uterus. Ann Anat Pathol 1926;3:697-718.

3. Cockshott WP, Hendrickse JP. Persistent arteriovenous fistulae following chemotherapy of malignant trophoblastic disease. Radiology 1967;88: 329-33.

4. Jain KA, Jeffrey RB Jr, Sommer FG. Gynecologic vascular abnormalities: diagnosis with Doppler US. Radiology 1991;178:549-51.

5. Fleming H, Östör AG, Pickel H, Fortune DW. Arteriovenous malformations of the uterus. Obstet Gynecol 1989;73:209-14.

6. Flynn MK, Levine D. The noninvasive diagnosis and management of a uterine arteriovenous malformation. Obstet Gynecol 1996; 88:650-2.

7. Jauniaux E. Ultrasound diagnosis and follow-up of a gestational trophoblastic disease. Ultrasound Obstet Gynecol 1998;11:367-77.

8. McLachlan MSF, Bird CC, Naiem EA, Scott JS. Uterine cirsoid aneurysm. Br J Obstet Gynaecol 1978;85:390-5.

9. Meilstrup JW, Fisher ME. Women's health case of the day: uterine arteriovenous malformation. AJR 1994;162:1457-8.

10. Tacchi D, Loose HW. Successful pregnancy after selective embolization of a post-molar vascular malformation. Case report. Br J Obstet Gynaecol 1988;95:814-7.

11. Ichikawa Y, Nakauchi T, Sato A, Oki A, Tsunoda H,
Yoshikawa H. Ultrasound diagnosis of uterine arteriovenous fistula associated with placental site trophoblastic tumor. Ultrasound Obstet Gynecol 2003;21:606-8.

12. Vogelzang RL, Nemcek AA Jr, Skrtic Z, Gorrell J, Lurain JR. Uterine arteriovenous malformations: primary treatment with therapeutic embolization. $\mathrm{J}$ Vasc Interv Radiol 1991;2:517-22.

13. Chapman DR, Lutz MH. Report of a successful delivery after nonsurgical management of a choriocarcinoma-related pelvic arteriovenous fistula. Am J Obstet Gynecol 1985;153:155-7.

14. Diwan RV, Brennan JN, Selim MA, et al. Sonographic diagnosis of arteriovenous malformation of the uterus and pelvis. J Clin Ultrasound 1983;11: 295-8.

15. Kelly FW. Forceps delivery after molar malignancy in a woman with arteriovenous malformation. A case report. J Reprod Med 2001;46:1013-6.

16 Chow TWP, Nwosu EC, Gould DA, Richmond DH. Pregnancy following successful embolisation of a uterine vascular malformation. Br J Obstet Gynaecol 1995;102:166-8.

17. Timmerman D, Wauters J, Van Callenbergh S, et al. Color Doppler imaging is a valuable tool for the diagnosis and management of uterine vascular malformations. Ultrasound Obstet Gynecol 2003;21: 570-7.

18. Joja I, Asakawa M, Motoyama K, et al. Uterine cirsoid aneurysm: MRI and MRA. J Comput Assist Tomogr 1996;20:290-4.

19. Cohen JM, Weinreb JC, Redman HC. Arteriovenous malformations of the extremities: MR imaging. Radiology 1986;158:475-9.

20. Fakhri A, Fishman EK, Mitchell SE, Siegelman SS, White RI. The role of CT in the management of pelvic arteriovenous malformations. Cardiovasc Intervent Radiol 1987;10:96-9.

21. Torres WE, Sones PJ Jr, Thames FM. Ultrasound appearance of a pelvic arteriovenous malformation. J Clin Ultrasound 1979;7:383-5. 